\title{
Empoderamiento femenino, emprendimiento y pobreza. El caso colombiano*
}

\author{
Women's empowerment, entrepreneurship and poverty. The Colombian case \\ Empoderamento feminino, empreendedorismo e pobreza. O caso colombiano
}

Jennifer Finke

Pontificia Universidad Javeriana, Colombia

ORCID: https://orcid.org/0000-0001-9957-962X

DOI: https://doi.org/10.11144/Javeriana.cao34.efepcc

Fabian Osorio-Tinoco

Pontificia Universidad Javeriana, Colombia

ORCID: https://orcid.org/0000-0002-0462-2398

Recibido: 05 Marzo 2021

Aceptado: 20 Septiembre 2021

Publicado: 30 Diciembre 2021

Fernando Pereira Laverde a

Pontificia Universidad Javeriana, Colombia

fpereira@javerianacali.edu.co

ORCID: https://orcid.org/0000-0002-8890-3858

\section{Resumen:}

Este artículo explora diferentes dimensiones del empoderamiento de las mujeres, que pueden desarrollarse durante el proceso emprendedor. Se evidencian cambios en las dimensiones conforme las mujeres avanzan en el desarrollo de sus emprendimientos, diferenciando características según el contexto (urbano o rural). Adicionalmente, muestra cuáles factores de empoderamiento son claves para la reducción de la pobreza. Se desarrolla un análisis de correspondencia múltiples y un modelo probit ordenado, con datos del Monitor Global de Emprendimiento (GEM por sus siglas en inglés) para Colombia. Los resultados permiten identificar las necesidades y los recursos disponibles para fortalecer el empoderamiento de las mujeres por medio del emprendimiento, mientras que la relación encontrada entre factores de empoderamiento económicos y los ingresos familiares aporta evidencia sobre la importancia del empoderamiento para la reducción de la pobreza.

Códigos JEL: J16, L26, M13.

Palabras clave: Empoderamiento, mujeres, emprendimiento, GEM, pobreza.

\section{Abstract:}

This paper explores the different dimensions of women's empowerment that can arise during the entrepreneurial process. It considers the changes in these dimensions as women advance in the development of their new ventures, differentiating characteristics according to the context (urban or rural). Another contribution refers to which empowerment factors developed by female entrepreneurs are key to reducing poverty. The proposal develops a multiple correspondence analysis and an ordered probit model, with data from the Global Entrepreneurship Monitor (GEM) Colombia. The results make it possible to identify what is needed to strengthen the empowerment of women through entrepreneurship, and the resources available for this. Furthermore, the relationship found between economic empowerment factors and family income provides evidence for the importance of empowerment for poverty reduction.

JEL Codes: J16, L26, M13.

Keywords: Empowerment, female, entrepreneurship, GEM, poverty.

\section{Resumo:}

Este artigo explora dimensões do empoderamento da mulher no âmbito do processo empreendedor e mostra se há mudanças nessas dimensões à medida que as mulheres avançam no desenvolvimento de seus empreendimentos, diferenciando características de acordo com o contexto (urbano ou rural). Além disso, contribui determinando quais fatores de empoderamento desenvolvidos por mulheres empresárias são fundamentais para reduzir a pobreza. Para a proposta, uma análise de correspondência múltipla e um modelo probit são desenvolvidos, com dados do Monitor de Empreendedorismo Global (GEM por suas siglas em inglês) para Colômbia. Os resultados permitem identificar as necessidades e recursos disponíveis para fortalecer o empoderamento das

Notas de autor

\footnotetext{
a Autor de correspondencia. E-mail: fpereira@javerianacali.edu.co
} 
mulheres, enquanto a relação encontrada entre os fatores de empoderamento econômico e a renda familiar evidencia a importância do empoderamento para a redução da pobreza.

Códigos JEL: J16, L26, M13.

Palavras-chave: Empoderamento, mulher, empreendedorismo, GEM, pobreza.

\section{Introducción}

El emprendimiento se ha convertido en un medio para aliviar la pobreza (Sutter, Bruton, \& Chen, 2019). Estudios realizados en Latinoamérica sobre emprendimientos desarrollados por mujeres dan cuenta de una relación entre precarias oportunidades -laborales y económicas- y el desarrollo de emprendimientos como una alternativa ante la desigualdad de género (Acosta, Zambrano, \& Suárez, 2017; Saavedra \& Camarena, 2015). Asimismo, han permitido identificar características distintivas de las emprendedoras y de sus negocios, así como limitaciones relacionadas con la posición de la mujer en la sociedad (Castiblanco, 2013; Ortiz, 2017).

Otros estudios se han enfocado en la relación del emprendimiento y el empoderamiento femenino, dado que el ejercicio del primero tiene un fuerte potencial para desarrollar y fortalecer procesos de empoderamiento en las mujeres: posibilita el acceso a recursos (materiales, humanos y sociales), el desarrollo de procesos comunitarios, la creación de mercados, favorece el desarrollo de una consciencia sobre las inequidades sociopolíticas entre hombres y mujeres, e impulsa la participación y el liderazgo de la mujer (Al-Dajani \& Marlow, 2013).

A pesar de que América Latina presenta una de las más altas tasas de emprendimiento femenino (Elam, Brush, Greene et al., 2019) y del potencial que tiene esto para reducir la pobreza (Korosteva \& StępieńBaig, 2020), las cifras de pobreza multidimensional y monetaria en Colombia muestran que las personas en hogares con jefatura femenina son más pobres que quienes pertenecen a hogares de jefatura masculina (DANE, 2019a, 2019b). Esto evidencia la necesidad de estudiar la relación entre emprendimiento y pobreza con una perspectiva de género, tomando en cuenta procesos importantes como el empoderamiento, que pueden desarrollarse en el avance de la actividad emprendedora.

En investigaciones previas el empoderamiento es un resultado del emprendimiento (Deedam \& Onoja, 2015; Fröcklin, Jiddawi, \& de la Torre-Castro, 2018; Huis Lensink, Vu et al., 2019; Khan, Kanwal, Nabi et al., 2016; Tahir, Kauser, Bury et al., 2018). Otros estudios exponen cómo los emprendimientos de las mujeres tienen el potencial para aportar a la reducción de la pobreza (Buendía \& Carrasco, 2013; Korosteva \& Stępień-Baig, 2020; Van der Zwan, Verheul, \& Thurik, 2012) demostrando que el contexto en el que se desarrolla el emprendimiento (urbano, rural), el tipo de emprendimiento (autoempleo, generadores de empleo) y su nivel de desarrollo (potencial, naciente, nuevo y establecido) son factores relevantes para la reducción de la pobreza y la brecha de género.

De lo anterior se infiere que existe un vacío en la literatura con respecto al análisis conjunto de emprendimiento, empoderamiento femenino y pobreza. Pocos son los estudios que consideran estas relaciones desde una perspectiva cuantitativa (p. ej. Buendía \& Carrasco, 2013). Este estudio es uno de los primeros en proponer una revisión de corte cuantitativo y basado en la data del Global Entrepreneurship Monitor (GEM) en Colombia, sobre la relación entre emprendimiento, empoderamiento femenino y la reducción de la pobreza.

La mirada sobre la relación entre emprendimiento y empoderamiento no debe remitirse de manera exclusiva al análisis de este último como un resultado, sino que el empoderamiento debe ser analizado en los diferentes momentos del proceso emprendedor. Este estudio considera la etapa de desarrollo del emprendimiento (nueva, naciente y establecida) y las diferencias de su contexto (urbano, rural) y de acuerdo con esto identifica factores de empoderamiento que aparecen significativos en cada etapa y son interpretados 
como logros o recursos de poder. Dado que el empoderamiento es un proceso dinámico y autoreforzante, los logros de poder en cada nivel pueden impulsar mayor empoderamiento e igualdad (Longwe, 1991) y esto puede ser rastreado en el desarrollo de un emprendimiento.

Los aportes de este estudio consisten en identificar los factores de empoderamiento con los que cuentan las mujeres en las actividades emprendedoras y evidenciar si existen cambios en estos según el avance de las mujeres en el desarrollo de su emprendimiento. Y además, determinar si existen diferencias entre los factores de empoderamiento según el contexto sea rural o urbano. Se enriquece así la literatura sobre emprendimiento femenino y empoderamiento al plantear factores de empoderamiento desarrollados en las emprendedoras que pueden ser determinantes para la reducción de la pobreza.

\section{Empoderamiento de la mujer emprendedora y la pobreza}

World Economic Forum mide la brecha global de género por medio de un índice compuesto de cuatro subíndices que examinan áreas críticas de desigualdad entre hombres y mujeres. Sus resultados indican que en promedio $68,6 \%$ de la población a nivel mundial ha alcanzado el balance de equidad. En el 31,4\% de la población en la cual persiste la brecha de desigualdad entre hombres y mujeres, las áreas que presentan un mayor desafío son las de empoderamiento político (24,7\%), y participación económica y oportunidad $(57,8 \%)$ (World Economic Forum, 2020). Este tipo de argumentaciones llevó a que la academia estudiara con detalle el empoderamiento de la mujer. Un área que ha recibido mucho interés es el empoderamiento como un resultado de las mujeres, específicamente a través del proceso emprendedor (Ahl, 2006).

En los estudios sobre la mujer y el género en el desarrollo, específicamente a un campo conocido como Mujer en el Desarrollo, el empoderamiento se presenta como un proceso de desafío de las relaciones de poder, lo que implica la obtención de mayor control sobre las fuentes, las cuales pueden ser bienes materiales o recursos intelectuales (Batliwala, 1997). Dada su naturaleza compleja y dinámica, el empoderamiento ha sido analizado desde diferentes dimensiones (Kabeer, 1999; Rowlands, 1997), niveles (Longwe, 1991) o componentes (Stromquist, 1997), esto ha dificultado tener consenso en la forma de medición, en la definición de indicadores y en los resultados de los estudios. Sin embargo, hay acuerdo en que el empoderamiento es un proceso e implica múltiples componentes en tanto "involucra conciencia, creencias y comportamientos individuales y colectivos integrados en la estructura social de contextos culturales específicos” (Huis, Hansen, Otten et al., 2017, p. 3).

Basados en lo anterior, este estudio propone tres dimensiones: individual, social y económica, en las que explora factores que son fundamentales para el empoderamiento, que pueden desarrollarse en diferentes etapas de la actividad emprendedora (naciente, nueva, establecida) y que están directamente relacionados con el emprendimiento. De esta manera es posible analizar, tanto las dimensiones de empoderamiento, como el proceso.

La dimensión individual ha sido conceptualizada y analizada como el sentido de autoconfianza y capacidad individual para ser agente de cambio en su propia vida, lo que implica ciertas creencias personales sobre sus acciones y los efectos o resultados que puede conseguir (Diener \& Biswas, 2005; Malhotra \& Schuler, 2005; Rowlands, 1995, 1997). Diferentes estudios han operacionalizado esta dimensión bajo la autoeficacia (Digan, Sahi, Mantok et al., 2019; Fröcklin et al., 2018; Malapit, Quisumbing, Meinzen-Dick et al., 2019; Huis et al., 2017).

La dimensión social implica una posición en la relación con el otro, que puede estar marcada por la influencia ejercida en dicha relación (Rowlands, 1995, 1997) e involucra creencias y acciones respecto a la posición de la mujer en relación con los demás (Huis et al., 2017). El estatus social se basa en roles sociales o en el cumplimiento de criterios socialmente valorados que afectan la capacidad para lograr objetivos (Uphoff, 2005). Esta dimensión ha sido operacionalizada en otros estudios bajo la participación en grupos (Alkire, 
Meinzen-Dick, Peterman et al., 2013; Malapit et al., 2019) o la percepción de reconocimiento por parte de la familia y la sociedad (Khan et al., 2016).

Por su parte, la dimensión económica ha sido la más estudiada, con indicadores objetivos como ingresos, propiedad de activos, perfiles de gastos y medidas subjetivas de control y autoridad en la toma de decisiones (Narayan, 2005). Implica el compromiso con una actividad productiva con algún grado de autonomía que brinde independencia económica y un mejor estatus (Stromquist, 1997), así como activos materiales, físicos o financieros, que permiten a las personas resistir ante eventos de crisis así como también ampliar el horizonte de elección (Narayan, 2005).

El emprendimiento implica un proceso de creación, desarrollo y sostenibilidad que facilita procesos de empoderamiento en la medida que permite a las mujeres el acceso a recursos, con la posibilidad de control sobre los mismos y genera un sentido de independencia y competencia. Asimismo habilita la posibilidad de asociación, colaboración y desarrollo de experiencia, lo que moviliza la confianza para el desarrollo de liderazgo mientras que los ingresos generados por sus actividades mejoran el bienestar de sí mismas, de sus familias y comunidades (Al-Dajani \& Marlow, 2013).

Algunos estudios se han centrado en evaluar la efectividad de los programas para empoderar a las emprendedoras (Fröcklin et al., 2018; Huis et al., 2019; Malapit et al., 2019). Sin embargo, la teoría del empoderamiento femenino y el emprendimiento deja ver que el análisis no es tan simple y que debe mirarse cómo se va dando el proceso de empoderamiento femenino en los diferentes momentos del proceso emprendedor, algo que no se ha estudiado lo suficiente.

Este estudio asume el empoderamiento y el emprendimiento como procesos distintos pero que se influencian o refuerzan mutuamente (Digan et al., 2019; Buendía \& Carrasco, 2013), y en este sentido, se propone indagar en cada etapa del proceso emprendedor cuáles son los factores de empoderamiento que se manifiestan en cada dimensión. Este es uno de los pocos estudios que aborda el empoderamiento femenino como un proceso que se da en el desarrollo de un emprendimiento y se manifiesta en logros o recursos de poder en las dimensiones individual, social y económica. Para este propósito se realiza un análisis de correspondencia con el fin de identificar cómo son las mujeres emprendedoras nuevas, nacientes y establecidas, en función de las tres dimensiones de empoderamiento adoptadas.

Además de estudiar el empoderamiento por medio del proceso emprendedor se mide su aporte a la reducción de la pobreza. Precisamente, existe una buena cantidad de investigaciones sobre el rol del emprendimiento en la reducción de la pobreza (Sutter et al., 2019) y del papel fundamental de la mujer y el emprendimiento (Korosteleva \& Stepien-Baig, 2020). Sin embargo, hay menos estudios cuantitativos enfocados en emprendimientos que midan si el empoderamiento femenino impulsa el desarrollo y aporta a la reducción de la pobreza (p. ej. Buendía \& Carrasco, 2013; San Vicente Portes, Atal, \& Juárez, 2019).

Diferentes autores que se han enfocado en el empoderamiento dejan claro el vínculo entre el desempoderamiento y la pobreza. En este sentido, el empoderamiento es "la expansión de los activos y las capacidades de las personas pobres para participar, negociar, influir, controlar y responsabilizar a las instituciones que afectan sus vidas" (Narayan, 2002, p. vi). Así, la insuficiencia de medios o recursos para satisfacer las necesidades básicas, restringe la posibilidad de contar con alternativas de elección, lo que limita el proceso de empoderamiento de las mujeres (Kabeer, 1999).

Adicionalmente, la reducción de la desigualdad por medio de la ampliación de las capacidades humanas y una mejor distribución de bienes o activos puede aportar a un mejor desempeño de las políticas y la inversión social cuando se logra que las personas pobres participen en los mercados más efectivamente (Narayan, 2002).

Estudios previos han demostrado que el empoderamiento de la mujer mejora la riqueza y el bienestar regional (Negash, 2006; Nadim \& Nurlukman, 2017) dado que ellas demuestran mayor voluntad de invertir sus ingresos en la familia y también en las comunidades, en comparación con los hombres, difundiendo mayor riqueza y bienestar. Entonces, las mujeres emprendedoras pondrán sus utilidades al servicio de sus familias con mayor voluntad generando una reducción de la pobreza a través de los ingresos familiares. En esta dirección, 
instituciones académicas y agencias para la cooperación internacional han apostado al empoderamiento femenino por medio del emprendimiento para el desarrollo (Global Training \& Development Institute, 2019; International Labour Organization, 2019; Mercy Corps, 2017). Por lo anterior, se formuló la siguiente hipótesis.

H1: El empoderamiento de las mujeres emprendedoras tiene una influencia en la reducción de la pobreza.

\section{Metodología}

\section{Base de datos y muestra}

La investigación utiliza los datos del Monitor Global de Emprendimiento (GEM por sus siglas en inglés) Colombia para el periodo comprendido entre los años 2015 y 2017. La base de datos permite identificar a las emprendedoras en Colombia y algunas variables pueden ser utilizadas como indicadores proxys de empoderamiento.

GEM con una muestra aleatoria y probabilística es representativa de la población adulta colombiana y garantiza la representación balanceada en función de las características del universo: edad (18-64 años), género (mujeres y hombres) y región (urbana y rural). Si bien esta diferenciación ha sido ampliamente discutida (Figueroa-Armijos \& Johnson, 2013; Lafuente, Vaillant, \& Rialp, 2007), este trabajo así como los antes mencionados retoma la comprensión propuesta por GEM de clasificar como región rural a municipios y zonas con menos de 10.000 habitantes.

Los datos comprenden un total de 7.853 registros entre 2015 y 2017, y mide en promedio 290 variables. Para el objetivo del estudio se seleccionaron los datos de mujeres emprendedoras colombianas de acuerdo con la variable tasa de actividad emprendedora $(\text { TEA })^{1}$, lo que limita el número a un total de 938 registros. De las 938 mujeres, 758 pertenecen a regiones urbanas y 180 a las rurales.

\section{Variables}

GEM define una categorización para las emprendedoras de acuerdo con el nivel de desarrollo del emprendimiento, esto se caracteriza por la variable TEA y clasifica a las mujeres en emprendedoras potenciales, nacientes, nuevas o establecidas. La tabla 1 describe las variables de GEM consideradas en el estudio como significativas para identificar las diferentes dimensiones del empoderamiento de las emprendedoras en las tres etapas de desarrollo del emprendimiento. 
TABLA 1

Variables medidas en GEM

\begin{tabular}{|c|c|c|c|}
\hline Variable & Pregunta de la encuesta & Respuesta & Código \\
\hline \multirow{4}{*}{$\begin{array}{l}\text { Ingresos } \\
\text { familiares }\end{array}$} & \multirow{4}{*}{$\begin{array}{l}\text { Según los siguientes niveles de } \\
\text { renta, indicar en cuál de ellos se } \\
\text { sitúa su hogar, combinando en una } \\
\text { sola cantidad lo que ganan todos sus } \\
\text { miembros de forma aproximada. }\end{array}$} & $<1$ SMMLV & 1 \\
\hline & & Entre 1 y 2 SMMLV & 2 \\
\hline & & $>2 \mathrm{y}<4$ SMMLV & 3 \\
\hline & & $>4$ SMMLV & 4 \\
\hline \multirow{2}{*}{ Autoeficacia } & \multirow{2}{*}{$\begin{array}{l}\text { ¿Tiene los conocimientos, } \\
\text { habilidades y experiencia necesarios } \\
\text { para iniciar un nuevo negocio? }\end{array}$} & $\mathrm{Si}$ & 1 \\
\hline & & No & 0 \\
\hline \multirow[b]{2}{*}{ Propiedad } & \multirow{2}{*}{$\begin{array}{l}\text { ¿Es usted actualmente, ya sea solo o } \\
\text { con otras personas, propietario de } \\
\text { una firma que ayuda a gestionar, un } \\
\text { autoempleado o desarrolla una } \\
\text { actividad en la que vende bienes o } \\
\text { servicios a otros? }\end{array}$} & $\mathrm{Si}$ & 1 \\
\hline & & No & 0 \\
\hline \multirow[b]{2}{*}{ Inversión } & \multirow{2}{*}{$\begin{array}{l}\text { En los últimos } 3 \text { años ¿usted ha } \\
\text { proporcionado personalmente } \\
\text { fondos para una empresa o negocio } \\
\text { puesta en marcha por otras personas } \\
\text { excluyendo cualquier tipo inversión } \\
\text { en bonos, acciones o fondos } \\
\text { comunes de inversión? }\end{array}$} & $\mathrm{Si}$ & 1 \\
\hline & & No & 0 \\
\hline \multirow{2}{*}{$\begin{array}{l}\text { Reconocimien- } \\
\text { to de ocupación }\end{array}$} & \multirow{2}{*}{$\begin{array}{l}\text { En su país, ¿la mayoría de la gente } \\
\text { considera que emprender un nuevo } \\
\text { negocio es una elección de carrera } \\
\text { profesional deseable? }\end{array}$} & $\mathrm{Si}$ & 1 \\
\hline & & No & 0 \\
\hline \multirow{2}{*}{$\begin{array}{l}\text { Reconocimien- } \\
\text { to de estatus de } \\
\text { ocupación }\end{array}$} & \multirow{2}{*}{$\begin{array}{l}\text { En su país, ¿aquellos que tienen } \\
\text { éxito en iniciar un nuevo negocio } \\
\text { tienen un alto nivel de estatus y } \\
\text { respeto? }\end{array}$} & $\mathrm{Si}$ & 1 \\
\hline & & No & 0 \\
\hline \multirow{4}{*}{$\begin{array}{l}\text { Nivel de } \\
\text { educación }\end{array}$} & \multirow{4}{*}{$\begin{array}{l}\text { ¿Cuál es el nivel educativo/estudios } \\
\text { más alto que ha completado usted? }\end{array}$} & Primaria & 1 \\
\hline & & Secundaria & 2 \\
\hline & & Técnico/profesional & 3 \\
\hline & & Posgrado & 4 \\
\hline \multirow{5}{*}{ Edad } & \multirow{5}{*}{ ¿Cuántos años tienes? } & $18-29$ & $1 \& 0$ en otro caso \\
\hline & & $30-39$ & $1 \& 0$ en otro caso \\
\hline & & $40-49$ & $1 \& 0$ en otro caso \\
\hline & & $50-59$ & $1 \& 0$ en otro caso \\
\hline & & $>=60$ & $1 \& 0$ en otro caso \\
\hline \multirow{3}{*}{ Núcleo familiar } & \multirow{3}{*}{$\begin{array}{l}\text { ¿Cuántas personas incluyéndole a } \\
\text { usted componen su hogar } \\
\text { actualmente? }\end{array}$} & $1-2$ personas & 1 \\
\hline & & 3-4 personas & 2 \\
\hline & & 50 más personas & 3 \\
\hline \multirow{2}{*}{ Región } & \multirow{2}{*}{ Región } & Rural & 1 \\
\hline & & Urbano & 0 \\
\hline
\end{tabular}

SMMLV = Salario Mínimo Mensual Legal Vigente de Colombia, US\$261/por mes.

Fuente: elaboración propia.

Para probar la hipótesis planteada sobre la influencia de las dimensiones del empoderamiento femenino en la pobreza se tomó como variable dependiente los ingresos familiares por ser uno de los indicadores frecuentemente utilizados para medir pobreza y desigualdad (CEPAL, 2018; Korosteva \& Stępień-Baig, 2020).

La tabla 2 describe las dimensiones de empoderamiento y clasifica las variables tomadas de GEM como logros o recursos de poder, entendidos como factores de empoderamiento.

TABLA 2

Definiciones conceptuales y operacionales de las variables

\begin{tabular}{|c|c|c|}
\hline Dimensión & Descripción & Variable \\
\hline $\begin{array}{l}\text { Empoderamiento } \\
\text { individual }\end{array}$ & $\begin{array}{l}\text { Elementos internos como la } \\
\text { confianza en sí misma y en } \\
\text { sus capacidades para ser } \\
\text { agente de cambio y obtener } \\
\text { los resultados deseados. }\end{array}$ & $\begin{array}{l}\text { Autoeficacia: la percepción que tiene } \\
\text { la mujer sobre las capacidades para } \\
\text { desarrollar su emprendimiento. }\end{array}$ \\
\hline \multirow{2}{*}{$\begin{array}{l}\text { Empoderamiento } \\
\text { social }\end{array}$} & \multirow{2}{*}{$\begin{array}{l}\text { Elementos que pueden dar } \\
\text { cuenta de una posición de } \\
\text { reconocimiento en relación } \\
\text { con los demás. }\end{array}$} & $\begin{array}{l}\text { Reconocimiento de ocupación: la } \\
\text { percepción que tiene la mujer sobre su } \\
\text { ocupación como emprendedora, como } \\
\text { un rol deseable en la sociedad. }\end{array}$ \\
\hline & & $\begin{array}{l}\text { Reconocimiento de estatus de } \\
\text { ocupación: la percepción que tiene la } \\
\text { mujer sobre el lugar de reconocimiento } \\
\text { y respeto que le da su rol como } \\
\text { emprendedora en la sociedad. }\end{array}$ \\
\hline \multirow{2}{*}{$\begin{array}{l}\text { Empoderamiento } \\
\text { económico }\end{array}$} & \multirow{2}{*}{$\begin{array}{l}\text { Implica el acceso a activos } \\
\text { materiales, físicos o } \\
\text { financieros que posibiliten la } \\
\text { generación y el control de los } \\
\text { ingresos. }\end{array}$} & $\begin{array}{l}\text { Propiedad: posee un activo reflejado en } \\
\text { un emprendimiento. }\end{array}$ \\
\hline & & $\begin{array}{l}\text { Inversión: capacidad de inversión en } \\
\text { otros negocios. }\end{array}$ \\
\hline
\end{tabular}

Fuente: elaboración propia. 


\section{Método}

En primer lugar, se caracterizó el empoderamiento de las emprendedoras colombianas en diferentes momentos del proceso emprendedor: nacientes, nuevas y establecidas. Se realizó un Análisis de Correspondencias Múltiple (ACM) que es una técnica exploratoria y descriptiva para identificar estructuras latentes, analizando categorías de variables cualitativas, con lo que se logra identificar relaciones no lineales entre dichas categorías. Además, el ACM no asume ningún tipo de supuesto estadístico, por cuanto se trata de un estudio descriptivo multivariante, lo que permite analizar los datos sin relaciones a priori, permitiendo construir grupos de los diferentes factores de empoderamiento en las mujeres emprendedoras nacientes, nuevas y establecidas, que permite una comparación tanto entre grupos como en un contexto rural o urbano.

En segundo lugar, para determinar la influencia del empoderamiento sobre la pobreza y demostrar la hipótesis planteada, se recurre a un modelo de regresión probit ordenado debido a que la variable dependiente (ingresos familiares) es una variable discreta con un conjunto de alternativas que son múltiples y mutuamente excluyentes.

Si se define la variable $Y$ : ingresos familiares, de respuesta múltiple ordenada, la cual toma valores $\{0,1,2, \ldots 4\}$. El modelo probit ordenado para $Y \mathrm{i}$, se puede estimar a partir de un modelo de variable latente que se expresa en la ecuación 1.

$$
Y_{i}^{*}=F\left(X_{i}^{\prime} \beta\right)+u_{i}
$$

Donde $X_{i}^{\prime}$ no contiene constante, $\beta$ contiene $k$ parámetros y $u_{i} \sim N(0,1)$ (Cameron $\&$ Trivedi, 2005).

El modelo probit ordenado se construye a partir de una función lineal de variables independientes, así como de un conjunto de puntos de corte que determinan la elección del individuo (Wooldridge, 2011). Las categorías de elección se pueden establecer en valores discretos ordenados de la variable observada $Y i$, las cuales se definen en la ecuación 2.

$$
Y_{i}=\left\{\begin{array}{rrr}
1 & \text { si } & Y_{i}^{*} \leq c_{1} \\
2 & \text { si } & c_{1} \leq Y_{i}^{*}<c_{2} \\
3 & \text { si } & c_{2} \leq Y_{i}^{*}<c_{3} \\
4 & \text { si } & Y_{i}^{*} \geq c_{3}
\end{array}\right.
$$

Con $\mathrm{c}_{1}, \mathrm{c}_{2}, \mathrm{c}_{3}$, valores umbrales o puntos de corte en los cuales la persona define la elección, donde las distancias entre las categorías son desconocidas. La elección entre las categorías se enmarca en la ecuación 3.

$$
\begin{array}{ll}
j=1 & \text { menos de 1 SMMLV } \\
j=2 & \text { entre 1 y 2 SMMLV } \\
j=3 & \text { más de 2 y menos de SMMLV } \\
j=4 & \text { más de 4 SMMLV }
\end{array}
$$

De esta manera se estima la probabilidad de ocurrencia de la variable dependiente (ingresos familiares) en alguna de las categorías dadas según el comportamiento de las variables explicativas (factores de empoderamiento).

\section{Resultados y discusión}

Esta sección se divide en dos apartados: el primero muestra el perfil de las mujeres emprendedoras obtenido mediante el análisis de correspondencia múltiple. Esta técnica permite relacionar las variables de estudio y caracterizar a las mujeres emprendedoras, tanto las nacientes, las nuevas y las establecidas para comprender como son, y que las identifica. En el segundo apartado se presentan los resultados de la hipótesis planteada en 
el estudio mediante el uso del análisis de regresión probit ordenado, que permite conocer la influencia de las variables elegidas sobre los ingresos familiares de las mujeres como una medida de pobreza.

\section{Caracterización de emprendedoras}

Para caracterizar a la emprendedora en función de los factores de empoderamiento con los que cuenta e identificar si existen cambios en estos factores en la medida que las mujeres desarrollan sus emprendimientos se utilizó el Análisis de Correspondencias Múltiples (ACM). En función del grado de asociación de las variables, la técnica permitió dividir a las 758 emprendedoras urbanas en tres grupos. Cada uno de los grupos de emprendedoras recoge las características comunes que las representan. Esto se refleja en la tabla 3 donde se identifica un perfil de las emprendedoras según la etapa de desarrollo de su emprendimiento, factores de empoderamiento con los que cuentan y características sociodemográficas.

TABLA 3

Caracterización de emprendedoras urbanas

\begin{tabular}{|c|c|c|c|}
\hline Indicador & Grupo 1 & Grupo 2 & Grupo 3 \\
\hline Tipo de emprendedora & Nacientes & Nuevas & Establecidas \\
\hline \multirow{3}{*}{ Edad (años) } & \multirow{3}{*}{$18-29$} & \multirow{2}{*}{$18-29$} & $40-49$ \\
\hline & & & $50-59$ \\
\hline & & $30-39$ & 60 o más \\
\hline \multirow{2}{*}{ Educación } & \multirow[b]{2}{*}{-} & Técnico/Profesional & Primaria \\
\hline & & Posgrado & Secundaria \\
\hline $\begin{array}{l}\text { Tamaño de la familia } \\
\text { (personas) }\end{array}$ & - & - & 5 o más \\
\hline \multirow{2}{*}{ Ingreso } & \multirow{2}{*}{$\begin{array}{l}\text { Entre } 1 \text { y } 2 \\
\text { SMMLV }\end{array}$} & Entre 2 y 4 SMMLV & $<1$ SMMLV \\
\hline & & > 4 SMMLV & Entre 1 y 2 SMMLV \\
\hline Propiedad & No & $\mathrm{Si}$ & $\mathrm{Si}$ \\
\hline Inversión & No & $\mathrm{Si}$ & No \\
\hline $\begin{array}{l}\text { Reconocimiento de } \\
\text { estatus de ocupación }\end{array}$ & $\mathrm{Si}$ & - & - \\
\hline $\begin{array}{l}\text { Reconocimiento de } \\
\text { ocupación }\end{array}$ & - & No & $\mathrm{Si}$ \\
\hline Autoeficacia & No & $\mathrm{Si}$ & - \\
\hline
\end{tabular}

Fuente: elaboración propia.

Las mujeres del primer grupo son emprendedoras nacientes, que llevan menos de tres meses en el desarrollo de su emprendimiento, y sus edades están en el rango de 18 a 29 años.

Los factores de empoderamiento económico negativos sugieren que a pesar de llevar al menos tres meses en el desarrollo del negocio, aún no consideran que represente un activo bajo su propiedad y no tienen capacidad de inversión. Sin embargo, el indicador de la dimensión social positivo representa la mejora del estatus y del respeto en la sociedad por tener un negocio. De acuerdo con Uphoff (2005) el estatus social o la posición según roles sociales o criterios valorados socialmente afectan la capacidad para lograr los objetivos, por lo tanto el reconocimiento y la posición social que consideran las mujeres alcanzarían con el emprendimiento, puede influir en la motivación y el compromiso con el desarrollo de su negocio, así como en sus metas propuestas.

La importancia del compromiso con la actividad emprendedora recae en que la propiedad de un activo productivo permite a las mujeres generar recursos económicos en vez de ser beneficiarias pasivas de estos, lo que habilita mayores alternativas de elección y la posibilidad de ejercer agencia (Longwe \& Clarke, 1997). Fröcklin et al. (2018) hallaron un aumento en la autoridad para la toma de decisiones en el hogar, atribuido principalmente al aumento de los ingresos del hogar por las actividades productivas de las mujeres.

El manejo de un negocio implica de manera constante la toma decisiones. La experiencia y los logros adquiridos por las mujeres en estas dinámicas pueden incidir en la confianza que desarrollan en sí mismas y en sus capacidades, como muestran los resultados de los estudios (Al-Dajani \& Marlow, 2013; Fröcklin 
et al., 2018). Esto cobra especial relevancia para las emprendedoras urbanas nacientes, dado que también se caracterizan por una baja autoconfianza para el desarrollo de sus emprendimientos.

Las emprendedoras del segundo grupo desarrollaron actividades en su emprendimiento por un periodo de tres a 42 meses, con edades entre los 18 y 39 años. Tienen un nivel educativo entre técnico/profesional y posgrado, e ingresos mayores a dos salarios mínimos mensuales legales vigentes (SMMLV). Estas emprendedoras nuevas urbanas son el grupo que se caracteriza por mayores factores de empoderamiento. Entendiendo el empoderamiento como un proceso que se da en el desarrollo de un emprendimiento, es posible interpretar que los factores de propiedad, inversión y autoeficacia positivos, han sido logros de poder alcanzados por las mujeres conforme avanzan en el proceso emprendedor.

Mientras las emprendedoras nacientes urbanas no confían en sus capacidades para el desarrollo de sus emprendimientos, las nuevas con una experiencia previa consideran tener los conocimientos y habilidades necesarias. La autoeficacia puede tener una relación con logros alcanzados en el desarrollo de sus emprendimientos, cómo la capacidad de inversión en otros negocios o incluso los altos ingresos familiares y el nivel educativo. De acuerdo con Narayan (2005) la autoeficacia está influenciada por un proceso iterativo en el que las personas se sorprenden por el éxito alcanzado en las tareas desarrolladas, obtienen mayor confianza y con ello asumen roles y desafíos cada vez mayores.

Las emprendedoras nuevas urbanas -a diferencia de las nacientes- refieren ser propietarias de un activo productivo y además tienen la capacidad de invertir sus recursos en el desarrollo de otros emprendimientos. Sin embargo, el poseer recursos y alternativas de elección no es suficiente para alcanzar los logros deseados, se requiere la capacidad de percibirse a sí misma como apta para ocupar espacios en la toma de decisiones (Rowlands, 1995) y la confianza en sí misma juega un papel fundamental en ello. "Las capacidades como la habilidad y la confianza permiten a una persona usar las cualidades disponibles de manera más efectiva, para así aumentar el poder, al aumentar la probabilidad de que se logren los resultados deseados" (Uphoff, 2005, p. 225).

En la dimensión social, mientras que las emprendedoras nacientes urbanas referían esperar alcanzar una posición de respeto y estatus con el éxito en el desarrollo de su negocio, para las emprendedoras nuevas urbanas este factor ya no es relevante, en cambio, aparece como significativo el reconocimiento de ocupación negativo. En este sentido, a pesar de los logros alcanzados en las dimensiones individual y económica, una vez las mujeres tienen el estatus de emprendedoras, consideran que su posición no es una elección profesional deseable socialmente. Esta percepción puede, no solo afectar la capacidad para alcanzar sus logros, también puede limitar o fortalecer su capacidad de influencia y participación en una esfera colectiva. Otros estudios revelan que los emprendimientos han facilitado el empoderamiento de las mujeres en diferentes niveles, sin embargo, no logran transformar las construcciones sociales estructurales que limitan la capacidad de poder o influencia de las mujeres y persisten los estereotipos de género (Alario \& Morales, 2016; Al-Dajani \& Marlow, 2013).

El grupo tres de mujeres establecidas ha desarrollado actividades emprendedoras por más de 42 meses, se caracterizan por ser mayores de 40 años y tener niveles de educación entre primaria-secundaria. Algo que caracteriza particularmente a este grupo es que el tamaño del hogar está definido entre cinco o más personas y presentan ingresos menores a dos SMMLV. Para las emprendedoras establecidas urbanas el factor de autoeficacia deja de ser significativo en comparación con las anteriores etapas. Se mantiene significativa la propiedad de un activo productivo como un logro alcanzado y el factor de reconocimiento de ocupación que era negativo para las emprendedoras nuevas, en las establecidas es representativo como positivo. Esto indica que las mujeres una vez han avanzado y construido un negocio que evidencia sostenibilidad y crecimiento perciben que su elección profesional es reconocida como deseable socialmente. En este sentido se puede decir que gozan de una posición de reconocimiento, lo cual es fundamental para lograr objetivos y asumir desafíos de mayor envergadura. 
Es el caso de los estudios de Al-Dajani y Marlow (2013) y Huis et al. (2017) los cuales refieren que el adquirir una posición social de reconocimiento impulsa a las mujeres a ser modelos de referencia y esto puede actualizar los objetivos más allá del bienestar personal y de la familia, al bienestar de las mujeres y de la comunidad en general. De esta manera, cuando la propietaria de un negocio logra una identidad positiva y confianza para tomar decisiones, aumenta la probabilidad de ocupar roles de liderazgo dentro de su familia y comunidad inmediata (Al-Dajani \& Marlow, 2013).

Ahora, para conocer cómo se caracterizan las emprendedoras rurales, mediante la misma técnica de ACM, se segmentó a las 180 emprendedoras rurales en tres grupos función del grado de asociación de las variables. La tabla 4 muestra los grupos resultantes y recoge las características comunes que definen cada grupo de emprendedoras rurales.

TABLA 4

Caracterización de emprendedoras rurales

\begin{tabular}{|c|c|c|c|}
\hline Indicador & Grupo 1 & Grupo 2 & Grupo 3 \\
\hline Tipo de emprendedora & Nacientes & Nuevas & Establecidas \\
\hline \multirow{2}{*}{ Edad (años) } & \multirow{2}{*}{$30-39$} & \multirow{2}{*}{ - } & $50-59$ \\
\hline & & & $>=60$ \\
\hline \multirow{2}{*}{ Educación } & Técnico/Profesional & \multirow{2}{*}{ Secundaria } & \multirow{2}{*}{ Primaria } \\
\hline & Posgrado & & \\
\hline Tamaño de la familia & - & - & - \\
\hline Ingreso & - & $\begin{array}{l}\text { Más de } 4 \\
\text { SMMLV }\end{array}$ & - \\
\hline Propiedad & No & $\mathrm{Si}$ & $\mathrm{Si}$ \\
\hline Inversión & - & - & - \\
\hline $\begin{array}{l}\text { Reconocimiento de estatus } \\
\text { de ocupación }\end{array}$ & - & - & $\mathrm{Si}$ \\
\hline $\begin{array}{l}\text { Reconocimiento de } \\
\text { ocupación }\end{array}$ & No & - & - \\
\hline Autoeficacia & $\mathrm{Si}$ & - & No \\
\hline
\end{tabular}

SMMLV = Salario Mínimo Mensual Legal Vigente de Colombia, US\$261/por mes

$(-)=$ El indicador no es representativo del grupo

Fuente: elaboración propia.

El grupo uno se conforma por emprendedoras nacientes y sus edades están entre los 30 y 39 años. Son emprendedoras que confían en sus capacidades para desarrollar un negocio y se caracterizan por haber alcanzado un nivel educativo medio-alto (técnico/profesional y posgrado). La educación aparece como un recurso valioso para el logro de autoeficacia, y aunque las mujeres no tienen experiencia en el desarrollo de un negocio, ni consideran que su posición como emprendedora es reconocida como algo deseable socialmente, alcanzar un nivel educativo medio-alto puede incidir en su autoconfianza y autoeficacia, aumentando la probabilidad de actuación para obtener mayores logros (Narayan, 2005), lo cual puede fortalecer el desarrollo del emprendimiento.

Las emprendedoras rurales del segundo grupo son emprendedoras nuevas que han desarrollado actividades en su negocio en un periodo de tres a 42 meses y cuentan con ingresos mayores a cuatro SMMLV. Para este grupo es significativo el factor de propiedad, lo que indica que en esta etapa de desarrollo del emprendimiento su negocio ya es considerado un activo. La propiedad de un activo como recurso productivo puede ser visto como un logro alcanzado, que habilita la toma de decisiones sobre el uso y control de los ingresos para su bienestar y el de su familia como refieren los estudios de Al-Dajani y Marlow (2013) y Fröcklin et al. (2018).

Las mujeres del tercer grupo son emprendedoras establecidas que han mantenido sus negocios por más de 42 meses. Son mujeres mayores de 50 años. Este grupo, a pesar de la experiencia en el desarrollo del emprendimiento, no alcanza la confianza en sus capacidades; es posible pensar nuevamente en la educación como un recurso importante para el logro de autoeficacia, dado que estas se caracterizan por el nivel educativo más bajo (primaria). El nivel educativo bajo en las mujeres rurales implica mayores dificultades para aplicar a convocatorias y acceder a programas de apoyo para el emprendimiento (Acosta et al., 2017), lo que limita las posibilidades de expandir su horizonte de acción.

En la dimensión social las emprendedoras establecidas rurales consideran que su posición como emprendedoras les da un lugar de reconocimiento en la sociedad, esa posición de reconocimiento que logran 
una vez han evidenciado la sostenibilidad de sus negocios, ofrece el potencial para convertirse en modelos de referencia en sus comunidades, como sugiere el estudio de Khan et al. (2016).

\section{Relación entre empoderamiento e ingresos familiares}

Para probar si el empoderamiento tiene influencia sobre la reducción del nivel de pobreza, se estimó un modelo probit ordenado con las variables definidas para las diferentes dimensiones del empoderamiento femenino en el total de la muestra. En la tabla 5 se muestra que solo el empoderamiento económico representado por la propiedad y la inversión, y la variable educación presentan una relación significativa con los ingresos familiares. Esto significa para las emprendedoras colombianas que el tener acceso a la propiedad de un emprendimiento aumenta la probabilidad de mejorar los ingresos familiares, disminuyendo así el nivel de pobreza. De igual forma, la posibilidad de hacer inversiones en otros emprendimientos y contar con un mayor nivel de educación aumentará la probabilidad de mejorar los ingresos familiares de las emprendedoras.

TABLA 5

Modelo de estimación probit ordenado para los ingresos familiares

\begin{tabular}{ll}
\hline \multicolumn{1}{c}{ Variable } & Coef. $\mathbf{P}$ \\
\hline Reconocimiento de ocupación & $-0,033$ \\
\hline Reconocimiento de estatus de ocupación & $-0,087$ \\
\hline Propiedad & $0,4022^{* *}$ \\
\hline Inversión & $0,5944^{* * *}$ \\
\hline Autoeficacia & 0,225 \\
\hline Edad & 0,061 \\
\hline Educación & $0,7522^{* *}$ \\
\hline Tamaño del hogar & $-0,007$ \\
\hline${ }^{* *} p<0,01 ;{ }^{*} \mathrm{p}<0,05$. &
\end{tabular}

Fuente: elaboración propia.

El sentido y la magnitud de estas variables influencian el nivel de ingreso familiar de las emprendedoras colombianas. En la tabla 6 se presenta un análisis marginal para mirar dicha influencia con mayor detalle; se incluyen solamente las variables que han sido significativas en el modelo de estimación probit.

TABLA 6

Efectos marginales de estimación probit ordenado

\begin{tabular}{cccccccccc}
\hline & \multicolumn{2}{c}{$\begin{array}{c}\text { Menos de 1 } \\
\text { SMMLV }\end{array}$} & \multicolumn{2}{c}{$\begin{array}{c}\text { Entre 1 y 2 } \\
\text { SMMLV }\end{array}$} & \multicolumn{2}{c}{$\begin{array}{c}\text { Más de 2 y menos } \\
\text { de 4 SMMLV }\end{array}$} & \multicolumn{2}{c}{$\begin{array}{c}\text { Más de 4 } \\
\text { SMMLV }\end{array}$} \\
\hline Variable & $\mathrm{dy} / \mathrm{dx}$ & $\mathrm{Z}$ & $\mathrm{dy} / \mathrm{dx}$ & $\mathrm{Z}$ & $\mathrm{dy} / \mathrm{dx}$ & $\mathrm{Z}$ & $\mathrm{dy} / \mathrm{dx}$ & $\mathrm{Z}$ \\
\hline Propiedad & $-0,024$ & $-2,71$ & $-0,075$ & $-3,05$ & 0,066 & 2,91 & 0,032 & 3,07 \\
Inversión & $-0,027$ & $-3,22$ & $-0,118$ & $-2,70$ & 0,084 & 3,29 & 0,062 & 2,28 \\
Educación & $-0,042$ & $-7,12$ & $-0,144$ & $-8,51$ & 0,122 & 8,06 & 0,064 & 8,18 \\
\hline
\end{tabular}

SMMLV = Salario Mínimo Mensual Legal Vigente de Colombia, US\$261.

Fuente: elaboración propia.

Los resultados de la tabla 6 muestran que si la propiedad en un emprendimiento aumenta se espera que la probabilidad de que una emprendedora colombiana perciba ingresos familiares menores de un SMMLV disminuya en 2,45\%. De igual forma, un aumento en la inversión en el emprendimiento por parte de la emprendedora, hace que la probabilidad de percibir ingresos menores de un SMMLV, disminuya en 2,76\%. En el caso del aumento del nivel educativo también hay una disminución de la probabilidad de percibir ingresos menores de un SMMLV del 4,2\%. El sentido de la influencia de estas variables cambia a positivo en niveles mayores de ingresos, esto quiere decir que las variables del empoderamiento económico (propiedad e inversión) tienen una influencia en la probabilidad de aumentar los ingresos familiares y por lo tanto en la disminución de los niveles de pobreza. Los mayores niveles de propiedad y de inversión aumentarán 
la probabilidad de que las mujeres emprendedoras colombianas perciban ingresos entre dos SMMVL y cuatro SMMLV. Para la variable educación el efecto es el mismo. Por lo tanto, además de la dimensión de empoderamiento económico, la educación sigue siendo un factor que aumenta la probabilidad de que las mujeres emprendedoras reciban ingresos familiares entre dos SMMVL y cuatro SMMLV.

Dado que para estimar las relaciones en el estudio se ha propuesto diferenciar las emprendedoras urbanas de las rurales para identificar posibles diferencias entre ellas, en la tabla 7 y 8 se presentan los valores de las estimaciones del modelo probit ordenado, discriminado por urbano/rural y los efectos marginales respectivamente.

TABLA 7

Modelo de estimación probit ordenado diferenciado por región

\begin{tabular}{lcc}
\hline \multicolumn{1}{c}{ Variable } & Coef. (urbanas) $\boldsymbol{p}$ & Coef. (rurales) $\boldsymbol{p}$ \\
\hline Reconocimiento de ocupación & $-0,033$ & 0,077 \\
Reconocimiento de estatus de ocupación & $-0,077$ & $-0,021$ \\
Propiedad & $0,307^{*}$ & $0,946^{* *}$ \\
Inversión & $0,545^{*}$ & 0,765 \\
Autoeficacia & 0,283 & 0,021 \\
Edad & 0,040 & 0,177 \\
Educación & $0,729^{* *}$ & $0,983{ }^{* *}$ \\
Tamaño del hogar & $-0,042$ & 0,254 \\
\hline$"$ p $<0,01{ }^{*}$ p $<0,05$. & &
\end{tabular}

Fuente: elaboración propia.

En la tabla 7 es posible ver que al diferenciar por región, la propiedad y la educación presentan una relación significativa con los ingresos familiares en urbanas y rurales, sin embargo, la variable de inversión que es significativa para las urbanas, no lo es para las rurales.

TABLA 8

Efectos marginales por región de estimación probit ordenado

\begin{tabular}{lccccccccc}
\hline & $\begin{array}{c}\text { Menos de 1 } \\
\text { SMMLV }\end{array}$ & \multicolumn{2}{c}{$\begin{array}{c}\text { Entre 1 y 2 } \\
\text { SMMLV }\end{array}$} & \multicolumn{2}{c}{$\begin{array}{c}\text { Más de 2 y menos } \\
\text { de 4 SMMLV }\end{array}$} & \multicolumn{3}{c}{$\begin{array}{c}\text { Más de 4 } \\
\text { SMMLV }\end{array}$} \\
\hline Variable & $\mathrm{dy} / \mathrm{dx}$ & $\mathrm{Z}$ & $\mathrm{dy} / \mathrm{dx}$ & $\mathrm{Z}$ & $\mathrm{dy} / \mathrm{dx}$ & $\mathrm{Z}$ & $\mathrm{dy} / \mathrm{dx}$ & $\mathrm{Z}$ \\
\hline $\begin{array}{l}\text { Emprendedoras } \\
\text { Urbanas }\end{array}$ & & & & & & & & \\
$\begin{array}{l}\text { Propiedad } \\
\text { Inversión }\end{array}$ & $-0,016$ & $-1,91$ & $-0,060$ & $-2,08$ & 0,047 & 2,00 & 0,029 & 2,11 \\
Educación & $-0,022$ & $-2,69$ & $-0,110$ & $-2,34$ & 0,069 & 2,88 & 0,064 & 1,99 \\
Emprendedoras & $-0,036$ & $-6,04$ & $-0,145$ & $-7,61$ & 0,109 & 6,91 & 0,072 & 7,48 \\
Rurales & & & & & & & & \\
Propiedad & $-0,086$ & $-2,34$ & $-0,116$ & $-2,95$ & 0,180 & 2,99 & 0,022 & 2,12 \\
Inversión & $-0,046$ & $-1,59$ & $-0,139$ & $-1,10$ & 0,157 & 1,27 & 0,028 & 0,88 \\
Educación & $-0,077$ & $-4,00$ & $-0,147$ & $-3,78$ & 0,198 & 4,61 & 0,026 & 2,57 \\
\hline \multicolumn{7}{c}{ Fuente: elaboración propia. } \\
& & & & & & &
\end{tabular}

Las tablas 7 y 8 muestran resultados similares a los de la muestra total. Con la excepción de la variable de inversión que no es significativa para el grupo de emprendedoras rurales. Esto puede tener relación con los programas de asistencia social o las remesas dirigidas a las mujeres rurales (Rogan, 2017; Sharaunga \& Mudhara, 2021). También puede estar relacionado con una menor capacidad para producir ingresos en los emprendimientos de mujeres rurales dado que enfrentan mayores limitaciones (Acosta et al., 2017; Khan et al., 2016; Jaka \& Shava, 2018).

Los resultados de este estudio refuerzan los hallazgos de las investigaciones que demuestran que el ingreso familiar en mujeres emprendedoras es el indicador que presenta avances más significativos en el proceso de empoderamiento (Fröcklin et al., 2018; Huis et al., 2017; Tahir et al., 2018). Igualmente, refuerza la línea de investigación en la cual las mujeres suelen destinar sus ingresos principalmente para el bienestar de sus hogares, (Acosta et al., 2017; Alario \& Morales, 2016; Castiblanco, 2013; Saavedra \& Camarena, 2015; Tahir et al., 2018). 


\section{Conclusiones, implicaciones y limitaciones}

Una de las principales obligaciones de los gobiernos debería ser "erradicar la pobreza en todas sus formas" (Naciones Unidas, 2015) vinculando a su población a participar de la riqueza del país. Comprensiones económicas clásicas enmarcadas en la concepción de "remediar" la falta de recursos de las emprendedoras, con el supuesto que al cambiar esta situación el emprendimiento "florecerá" (Sutter et al., 2019), han simplificado el problema (Amorós \& Cristi, 2011) centrándose en evaluar y buscar como única solución el acceso más amplio a recursos económicos para las mujeres urbanas y rurales o a la propiedad de la tierra. Pero este supuesto no es siempre válido, pues toda acción emprendedora además de recursos, requiere una persona capaz para tomar acción emprendedora.

Para ampliar la comprensión del proceso emprendedor en las mujeres, por medio de un Análisis de Correspondencias Múltiples (ACM), este estudio caracterizó a las emprendedoras colombianas revelando factores de empoderamiento con los que contaron en el proceso. De esta manera fue posible tener una imagen de los diferentes momentos del proceso emprendedor y esto evidenció cómo las necesidades y recursos de las emprendedoras (urbanas y rurales), cambiaron durante el proceso emprendedor. Por otro lado, dada la importancia del empoderamiento para el alivio de la pobreza, el modelo probit ordenado consideró las dimensiones de empoderamiento para medir la relación con los ingresos familiares de las mujeres.

El modelo probit ordenado mostró una relación significativa entre las variables educación e ingresos familiares, tanto en las regiones rurales y urbanas, donde el conocimiento se convierte en un factor preponderante para la disminución de la pobreza. Igualmente se evidenció una relación significativa del empoderamiento económico y los ingresos familiares, tanto en los espacios rurales como urbanos.

El ACM evidenció que el reconocimiento social de las emprendedoras urbanas cambió según el avance en cada etapa. Si bien las emprendedoras nacientes urbanas se caracterizan por ser jóvenes, que consideran que el éxito en el desarrollo del emprendimiento les brinda estatus y respeto, no se identifican a sí mismas con confianza en sus capacidades para emprender. Cuando pasan al segundo nivel (emprendedoras nuevas) ese impulso inicial cambia y al enfrentarse al desarrollo del emprendimiento no consideran que este sea visto como una elección profesional deseable, pues es común escuchar que no les es fácil negociar con otros emprendedores hombres que no las reconocen como sus pares (Warren, 2004). Sin embargo, han logrado nuevos hitos en términos de la propiedad sobre nuevos recursos económicos y su capacidad de invertirlos, por lo que están más empoderadas con autoeficacia por los logros obtenidos en su recorrido.

En el último nivel del proceso emprendedor, las mujeres emprendedoras establecidas urbanas, que se caracterizan por contar con la propiedad de recursos económicos, son reconocidas socialmente como propietarias de sus empresas, a pesar de tener un nivel de educación bajo y ser de edad mayor.

La situación cambia para el proceso emprendedor femenino en las zonas rurales. En la etapa naciente se evidencia que inician el proceso más tarde, dado que las edades oscilan entre los 30 y 39 años, mientras que en las zonas urbanas las emprendedoras inician entre los 18 y 29 años. Si bien se caracterizan en la zona rural porque las mujeres que emprenden tienen un mayor nivel educativo frente al grupo urbano, no reciben el reconocimiento social de su contexto cercano, y a pesar de no contar con la propiedad de los recursos sí cuentan con autoeficacia que es un recurso importante en estas etapas iniciales emprendedoras. En la siguiente etapa las emprendedoras rurales nuevas se caracterizan por tener propiedad, lo que les permite mostrar un mayor nivel de ingresos. Las emprendedoras rurales establecidas, que están en la tercera etapa, se caracterizan por ser mayores de 50 años, con nivel educación bajo, pero con reconocimiento social y la propiedad de un activo productivo, se diferencian de otros grupos por no tener autoeficacia.

Los resultados sugieren que el nivel educativo alcanzado por las emprendedoras puede tener alguna relación con el desarrollo de autoeficacia; las emprendedoras que se caracterizan por un nivel educativo alto (nacientes rurales y nuevas urbanas) también se caracterizan por autoeficacia, mientras que las emprendedoras establecidas rurales que se caracterizan por el nivel educativo menor (primaria) refieren ausencia de 
autoeficacia. Aunque Van Dinther, Dochy, \& Segers (2011) muestran que los programas de educación superior pueden influir en la autoeficacia de los estudiantes, esta posible relación requiere de una mayor exploración en el caso de las mujeres emprendedoras.

Identificar las dimensiones y los factores de empoderamiento es útil para dirigir intervenciones más efectivas que permitan aprovechar las fortalezas o logros alcanzados en cada etapa de desarrollo del emprendimiento. Así, por medio del proceso emprendedor es posible aprovechar el potencial de transformar la posición de la mujer. Se resalta también la importancia de apoyar el desarrollo de emprendimientos en mujeres que inician sus negocios, para el fortalecimiento y sostenibilidad de estos, dado que fue posible comprobar para las emprendedoras colombianas que la propiedad de un emprendimiento aumenta la probabilidad de mejorar los ingresos familiares y, por ende, aporta a disminuir la pobreza.

Para los grupos de emprendedoras urbanas y rurales nacientes se recomienda fortalecer el desarrollo de sus emprendimientos facilitando el acceso a mercados (Fletschner \& Mesbah, 2011), créditos (Khan et al., 2016) o subvenciones (De Mel, McKenzie \& Woodruff, 2014) para un alcance progresivo de empoderamiento económico. Por otro lado, la generación de procesos de valor agregado puede ayudar a establecer relaciones sólidas con los agentes del mercado, facilitando su integración al mismo, especialmente para las mujeres rurales (Orozco \& Baldrich, 2020). Para las nacientes urbanas que no refieren autoeficacia a diferencia de las nacientes rurales, se considera pertinente fortalecer la formación educativa (formal e informal) y el acceso a capacitación técnica (Fröcklin et al., 2018), para el avance progresivo en el empoderamiento individual. Ahora, las nacientes urbanas debido a que son mujeres jóvenes (18-39 años) con reconocimiento social, es relevante aprovechar este reconocimiento con su participación en grupos de emprendedoras, para la generación de influencia como modelos de referencia (Al-Dajani \& Marlow, 2013).

Debido a que las emprendedoras nacientes rurales cuentan con autoeficacia, con altos niveles educativos, pero hay una ausencia de reconocimiento social, la participación o conformación de grupos o redes de apoyo de emprendedoras puede motivar y desarrollar el liderazgo para compartir sus conocimientos (Warren, 2004), y también la participación en colectividades o asociaciones de mujeres puede fortalecer sus habilidades y facilitar la integración al mercado (Orozco \& Baldrich, 2020). Los grupos de emprendedoras urbanas y rurales establecidas requieren también un apoyo en formación educativa para fortalecer la autoeficacia, sin embargo se debe tener en cuenta la diferencia de los rangos de edad (40-49, 50-59 y mayores de 60 años) y los conocimientos y recursos previos con los que cuentan (Acosta et al., 2017). Específicamente para las rurales establecidas, que cuentan con reconocimiento social por su ocupación, el reconocimiento de sus saberes y experiencia previa puede legitimarlos y fortalecer la autoeficacia.

De acuerdo con los resultados obtenidos y los referentes teóricos revisados es posible decir que los logros de poder en cada dimensión deben ser la base para impulsar el empoderamiento en las otras dimensiones. En las diferentes etapas es posible ver cómo algunos recursos se expanden, mientras que otros dejan de ser significativos. Para avanzar en el proceso de empoderamiento se hace necesario aprovechar los logros ya alcanzados en cada dimensión, para configurar un conjunto de recursos (económicos, sociales y materiales) que permitan mayor capacidad de decisión con el fin de alcanzar los logros necesarios en función de los intereses de las mujeres (Kabeer, 1999).

Estos resultados muestran la diversidad emprendedora femenina en las diferentes etapas. Esta riqueza indicada por el uso de dos herramientas estadísticas evidencian la necesidad de ajustar innovadoramente los programas de apoyo a las mujeres emprendedoras. Esta comprensión brinda la identificación de variables claves para investigaciones futuras y sugiere ampliar los indicadores de empoderamiento con el diseño y validación de escalas para resultados más precisos. Para ampliar la caracterización de cada etapa y determinar el comportamiento de los indicadores de empoderamiento se sugiere un estudio longitudinal que reflejen las dinámicas que viven las emprendedoras pasando por cada etapa. Por último, otra limitación importante de este estudio surge de la naturaleza del instrumento utilizado para la recolección de la información, específicamente al apoyarse en la autopercepción reportada por cada mujer encuestada. 


\section{Consideraciones éticas}

Dada la naturaleza del estudio no requirió de aval ético. Se tuvieron en cuenta las normas de citación correspondientes a derechos de autor y todos los autores responden por la precisión y originalidad del texto completo.

\section{Contribución de los autores}

Los autores contribuyeron en igual proporción en el diseño, la metodología, el análisis de resultados y la composición de este artículo.

\section{Financiación}

Este trabajo fue financiado por el Proyecto No. 1036 de Investigar "Caracterización de las actividades emprendedoras que buscan empoderar a las mujeres rurales del Valle del Cauca" de la convocatoria interna 2018 de la Vicerrectoría Académica de la Pontificia Universidad Javeriana Cali, Colombia.

\section{Conflictos de interés}

Los autores declaran no tener ningún tipo de conflicto de interés.

\section{Referencias}

Acosta, B., Zambrano, S., \& Suárez, M. (2017). Emprendimiento femenino y ruralidad en Boyacá, Colombia. Criterio Libre, 15(26), 216-236. https://doi.org/10.18041/1900-0642/criteriolibre.2017v15n26.1048

Ahl, H. (2006). Why research on women entrepreneurs needs new directions. Entrepreneurship Theory and Practice, 30(5), 595-621. https://doi.org/10.1111/j.1540-6520.2006.00138.x

Alario, M., \& Morales, E. (2016). Iniciativas de las mujeres: emprendimiento y oportunidades en el espacio rural de Castilla y León. Documents d’Anàlisi Geogràfica, 62(3), 613-637. http://dx.doi.org/10.5565/rev/dag.369

Al-Dajani, H., \& Marlow, S. (2013). Empowerment and entrepreneurship: A theoretical framework. International Journal of Entrepreneurial Behavior and Research, 19(5), 503-524. https://doi.org/10.1108/IJEBR-10-2011-0 138

Alkire, S., Meinzen-Dick, R., Peterman, A., Quisumbing, A., Seymour, G., \& Vaz, A. (2013). The women's empowerment in agriculture index. World Development, 52, 71-91. https://doi.org/10.1016/j.worlddev.2013. 06.007

Amorós, J., \& Cristi, O. (2011). Poverty and entrepreneurship in developing countries. En: M. Minniti (Ed.), The Dynamics of Entrepreneurship: Evidence from Global Entrepreneurship Monitor Data (pp. 209-230). Oxford: Oxford University Press. https://doi.org/10.1093/acprof:oso/9780199580866.001.0001

Batliwala, S. (1997). El significado del empoderamiento de las mujeres: nuevos conceptos desde la acción. En M. León (Ed.), Poder y empoderamiento de las mujeres (pp. 187-211). Bogotá: Tercer Mundo.

Buendía, I. \& Carrasco, I. (2013). Mujer, actividad emprendedora y desarrollo rural en América Latina y el Caribe. Cuadernos de Desarrollo Rural, 10(72) 21-45. https://doi.org/10.11144/Javeriana.cdr10-72.maed

Cameron, C., \& Trivedi, P. (2005). Microeconometrics: Methods and applications. New York: Cambridge University Press. https://doi.org/10.1017/CBO9780511811241 
Castiblanco, S. (2013). La construcción de la categoría de emprendimiento femenino. Revista Facultad de Ciencias Económicas, 21(2), 53-66. https://doi.org/10.18359/rfce.656

Comisión Económica para América Latina y el Caribe - CEPAL (2018). La ineficiencia de la desigualdad. Santiago de Chile: Naciones Unidas. http://hdl.handle.net/11520/23424

Deedam, N. J., \& Onoja, A. O. (2015). Impact of Poverty Alleviation Programmes on Indigenous Women's Economic Empowerment in Nigeria Evidence from Port harcourt Metropolis. Consilience, 14(2), 90-105. https://doi.org /10.7916/consilience.v0i14.4682

De Mel, S., McKenzie, D., \& Woodruff, C. (2014). Business training and female enterprise start-up, growth, and dynamics: experimental evidence from Sri Lanka. J. Journal of Development Economics, 106, 199-210. https://d oi.org/10.1016/j.jdeveco.2013.09.005

Departamento Administrativo Nacional de Estadística - DANE (2019a). Boletín Técnico. Pobreza Monetaria en Colombia Año 2018. Recuperado el 16 de agosto de 2019, de https://www.dane.gov.co/files/investigaciones/c ondiciones_vida/pobreza/2018/bt_pobreza_monetaria_18.pdf

Departamento Administrativo Nacional de Estadística DANE (2019b). Boletín Técnico. Pobreza Multidimensional en Colombia Año 2018. Recuperado el 16 de agosto de 2019, de https://www.dane.gov.co/files/investigaciones/co ndiciones_vida/pobreza/2018/bt_pobreza_multidimensional_18.pdf

Diener, E., \& Biswas, R. (2005). Psychological Empowerment and Subjective Well-Being. En D. Narayan (Ed.), Measuring Empowerment. Cross-Disciplinary Perspectives (pp. 125-140). Washington D.C.: The World Bank. h ttp://hdl.handle.net/10986/7441

Digan, S., Sahi, G., Mantok, S., \& Patel, P. (2019). Women's perceived empowerment in entrepreneurial efforts: The role of bricolage and psychological capital. Journal of Small Business Management, 57(1), 206-229. https://do i.org/10.1111/jsbm.12402

Elam, A. B., Brush, C. G., Greene, P. G., Baumer, B., Dean, M. \& Heavlow, R. (2019). Global Entrepreneurship Monitor 2018/2019. Women's Entrepreneurship Report. London: Global Entrepreneurship Research Association, London Business School.

Figueroa-Armijos, M., \& Johnson, T. (2013). Entrepreneurship in Rural America Across Typologies, Gender and Motivation. Journal of Developmental Entrepreneurship, 18(2), 1-37. https://doi.org/10.1142/S10849467135 00143

Fletschner, D., \& Mesbah, D. (2011). Gender disparity in access to information: Do spouses share what they know? World Development, 39(8), 1422-1433. https://doi.org/10.1016/j.worlddev.2010.12.014

Fröcklin, S., Jiddawi, N., \& de la Torre-Castro, M. (2018). Small-scale innovations in coastal communities: Shellhandicraft as a way to empower women and decrease poverty. Ecology and Society, 23(2). https://doi.org/10.5 751/ES-10136-230234

Global Training \& Development Institute (2019). Empowering Women through Social Entrepreneurship: Fellowship Exchange with U.S.-Costa Rica; Economic Empowerment Program-Costa Rica, Columbia, Peru and Panama. Recuperado el 11 de agosto de 2019 de https://gtdi.uconn.edu/about/

Huis, M., Hansen, N., Otten, S., \& Lensink, R. (2017). A three-dimensional model of women's empowerment: Implications in the field of microfinance and future directions. Frontiers in Psychology, 8(1678). https://doi.or g/10.3389/fpsyg.2017.01678

Huis, M., Lensink, R., Vu, N., \& Hansen, N. (2019). Impacts of the Gender and Entrepreneurship Together Ahead (GET Ahead) training on empowerment of female microfinance borrowers in Northern Vietnam. World Development, 120, 46-61. https://doi.org/10.1016/j.worlddev.2019.04.001

International Labour Organization (2019). Empower: Building peace through the economic empowerment of women in Northern Sri Lanka. https://www.ilo.org/colombo/whatwedo/projects/WCMS_614448/lang--en/index.htm 1 .

Jaka, H., \& Shava, E. (2018). Resilient rural women's livelihoods for poverty alleviation and economic empowerment in semi-arid regions of Zimbabwe. Jamba-Journal of Disaster Risk Studies 10(1), 524. https://doi.org/10.4102 /jamba.v10i1.524 
Kabeer, N. (1999). Resources, agency, achievements: Reflections on the measurement of women's empowerment. Development and Change, 30(3), 435-464. https://doi.org/10.1111/1467-7660.00125

Khan, M. A., Kanwal, N., Nabi, P. G., \& Shah, A. A. (2016). Empowering women entrepreneurs through microcredit: Assessing the role of Sarhad Rural Support Program in Pakistan. Journal of Entrepreneurship Management and Innovation, 12(1), 91-112. https://doi.org/10.7341/20161215

Korosteva, J., \& Stępień-Baig, P. (2020). Climbing the poverty ladder: The role of entrepreneurship and gender in alleviating poverty in transition economies. Entrepreneurship \& Regional Development, 32(1-2), 197-220. http s://doi.org/10.1080/08985626.2019.1640482

Lafuente, E., Vaillant, Y., \& Rialp, J. (2007). Regional differences in the influence of role models: Comparing the entrepreneurial process of rural Catalonia. Regional Studies, 41(6), 779-795. https://doi.org/10.1080/003434 00601120247

Longwe, S. (1991). Gender Awareness: The Missing Element in the Third World Development Project. En T. Wallace, \& C. March (Eds.), Changing Perceptions: Writings on Gender and Development (pp. 149-157). Oxford: OXFAM.

Longwe, S., \& Clarke, R. (1997). El marco conceptual de igualdad y empoderamiento de las mujeres. En M. León (Ed.), Poder y empoderamiento de las mujeres. (pp. 173-186). Bogotá: Tercer Mundo.

Malapit, H., Quisumbing, A., Meinzen-Dick, R., Seymour, G., Martinez, E., Heckert, J., Rubin, D., Vaz, A. \& Yount, K. (2019). Development of the project-level Women's Empowerment in Agriculture Index (pro-WEAI). World Development 122, 675-692. https://doi.org/10.1016/j.worlddev.2019.06.018

Malhotra, A., \& Schuler, S. (2005). Women's empowerment as a variable in international development. En D. Narayan (Ed.), Measuring Empowerment. Cross-Disciplinary Perspectives (pp.71-88). Washington D.C.: The World Bank. http://hdl.handle.net/10986/7441

Mercy Corps (2017). Mujeres rurales del Cauca. Recuperado el 8 de agosto de 2019, de https://mercycorps.org.co/p rograma/mujeresrurales-del-cauca.

Naciones Unidas (2015). Sustainable Development Goals. Recuperado, de https://www.undp.org/content/undp/es/ home/sustainable-development-goals/goal-1-no-poverty.html

Nadim, S., \& Nurlukman, A. (2017). The impact of women empowerment on poverty reduction in rural area of Bangladesh: Focusing on village development program. Journal of Government and Civil Society, 1(2), 135-157. http://dx.doi.org/10.31000/jgcs.v1i2.444

Narayan, D. (2002). Empowerment and Poverty Reduction: A Sourcebook. Washington D.C.: World Bank. http://hd l.handle.net/10986/15239

Narayan, D. (2005). Conceptual Framework and Methodological Challenges. En D. Narayan (Ed.), Measuring Empowerment. Cross- Disciplinary Perspectives (pp. 3-38). Washington D.C.: The World Bank. http://hdl.han dle.net/10986/7441

Negash, T. (2006). Education in Ethiopia: From crisis to the brink of collapse. Stockholm: Nordiska Afrikainstitutet.

Orozco, L., \& Baldrich, V. (2020). Gender, violence and social resources in rural producers' economic decisions.Journal of Rural Studies, 80, 222-234. https://doi.org/10.1016/j.jrurstud.2020.09.011

Ortiz, P. (2017). El discurso sobre el emprendimiento de la mujer desde una perspectiva de género. Vivat Academia, 140, 115-129. https://doi.org/10.15178/va.2017.140.115-129

Rogan M. (2017). Food poverty, hunger and household production in rural Eastern Cape households. Development Southern Africa 35(1), 90-104. https://doi.org/10.1080/0376835X.2017.1358602

Rowlands, J. (1995). Empowerment examined. Development in Practice,5(2), 101-107. https://doi.org/10.1080/096 1452951000157074

Rowlands, J. (1997). Questioning empowerment: Working with women in Honduras. Oxford: Oxfam.

Saavedra, M., \& Camarena, M. (2015). Retos para el emprendimiento femenino en América Latina. Criterio Libre, 13(22), 129-152. https://doi.org/10.18041/1900-0642/criteriolibre.2015v13n22.129 
San Vicente Portes, L., Atal, V., \& Juárez, M. (2019). From households to national statistics: Macroeconomic effects of Women's empowerment. Economic Modelling, 79, 286-294. https://doi.org/10.1016/j.econmod.2019.01.024

Sharaunga, S., \& Mudhara, M. (2021). Analysis of Livelihood Strategies for Reducing Poverty among Rural Women's Households: A Case Study of KwaZulu-Natal, South Africa. Journal of International Development, 33(1), 127-150. https://doi.org/10.1002/jid.3516

Stromquist, N. (1997). La búsqueda del empoderamiento: En qué puede contribuir el campo de la educación. En M. León (Ed.), Poder y empoderamiento de las mujeres (pp. 173-186). Bogotá: Tercer Mundo.

Sutter, C., Bruton, G., \& Chen, J. (2019). Entrepreneurship as a solution to extreme poverty: A review and future research directions. Journal of Business Venturing, 34(1), 197-214. https://doi.org/10.1016/j.jbusvent.2018.06 .003

Tahir, W., Kauser, R., Bury, M., \& Bhatti, J. (2018). 'Individually-led' or 'female-male partnership' models for entrepreneurship with the BISP support: The story of women's financial and social empowerment from Pakistan. Women's Studies International Forum 68, 1-10. https://doi.org/10.1016/j.wsif.2018.01.011

Uphoff, N. (2005). Analytical issues in measuring empowerment at the community and local levels. En D. Narayan (Ed.), Measuring empowerment. cross-disciplinaryperspectives (pp. 219-246). Washington D.C.: The World Bank. http://hdl.handle.net/10986/7441

Warren, L. (2004). Negotiating entrepreneurial identity: Communities of practice and changing discourses. International Journal of Entrepreneurship and Innovation 5(1), 25-35. https://doi.org/10.5367/00000000477 2913764

Wooldridge, J. (2011). Econometric Analysis of Cross Section and Panel Data, $2^{\text {nd }}$ ed. Cambridge MA: The MIT Press. World Economic Forum. (2020). Global Gender Gap Report 2020. Ginebra: World Economic Forum.

Van der Zwan, P., Verheul, I., \& Thurik, A. R. (2012). The entrepreneurial ladder, gender, and regional development. Small Business Economics, 39(3), 627-643. https://doi:10.1007/s11187-011-9334-7

Van Dinther, M., Dochy, F., \& Segers, M. (2011). Factors affecting students' self-efficacy in higher education. Educational Research Review, 6(2), 95-108. https://doi.org/10.1016/j.edurev.2010.10.003

\section{Notas}

* Artículo de investigación.

1 La TEA clasifica a las emprendedoras entre emprendedoras potenciales (quienes reconocen oportunidades y cuentan con conocimientos y habilidades en emprendimientos), emprendedoras nacientes (quienes inician una actividad emprendedora pagando salarios a sí misma o a empleados por un periodo menor a tres meses) emprendedoras nuevas o en desarrollo (quienes por un tiempo de 3 a 42 meses han estado en su actividad emprendedora pagando salarios) y emprendedoras establecidas (quienes por más de 42 meses han pagado salarios, evidenciando persistencia, sostenibilidad y crecimiento).

\section{Licencia Creative Commons CC BY 4.0}

Sugerencia de citación: Finke, J., Osorio-Tinoco, F., \& Pereira, F. (2021). Empoderamiento femenino, emprendimiento y pobreza. El caso colombiano. Cuadernos de Administración, 34. https://doi.org/10.111 44/Javeriana.cao34.efepcc 